\title{
La représentation : point focal de l'apprentissage
}

\author{
Lucie Sauvé et Louis Machabée
}

\section{(2) OpenEdition}

Journals

Édition électronique

URL : https://journals.openedition.org/ere/6859

DOI : 10.4000/ere.6859

ISSN : 2561-2271

Éditeur

Centr'ERE

\section{Référence électronique}

Lucie Sauvé et Louis Machabée, «La représentation : point focal de l'apprentissage », Éducation relative à l'environnement [En ligne], Volume 2 | 2000, mis en ligne le 15 septembre 2000, consulté le 21 septembre 2021. URL : http://journals.openedition.org/ere/6859 ; DOI : https://doi.org/10.4000/ere 6859

Ce document a été généré automatiquement le 21 septembre 2021.

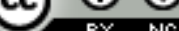

La revue Éducation relative à l'environnement est mise à disposition selon les termes de la Licence Creative Commons Attribution - Pas d'Utilisation Commerciale 4.0 International. 


\title{
La représentation : point focal de l'apprentissage
}

\author{
Lucie Sauvé et Louis Machabée
}

1 Dans le cadre d'une démarche de recherche-intervention en éducation relative à l'environnement, menée en collaboration avec un groupe d'enseignants du secondaire, nous nous sommes intéressés à l'analyse des représentations de ces derniers comme stratégie d'évaluation de leurs apprentissages en cours de recherche. La représentation constitue, en effet, le point focal des apprentissages relatifs à un objet. L'analyse diachronique d'une représentation nous informe sur la synthèse progressive que fait le sujet de ses nouvelles expériences relatives à cet objet (expériences d'ordre cognitif, affectif et du domaine de l'agir) et peut aussi donner des indications sur le processus de construction des schèmes « représentationnels ».

2 Nous rappellerons d'abord les principaux éléments du cadre théorique sur lequel s'appuie notre stratégie évaluative. Nous présenterons ensuite l'essentiel de la démarche menée avec les enseignants et nous identifierons les techniques de collecte de données évaluatives que nous avons adoptées. Enfin, nous exposerons et commenterons certains résultats obtenus.

La représentation d'un objet (il s'agit ici essentiellement de l'éducation relative à l'environnement) correspond à un ensemble de conceptions, d'attitudes, de valeurs, de significations, de connotations, d'associations, et autres éléments d'ordre cognitif ou affectif qui à la fois résultent de l'expérience de cet objet et déterminent la relation du sujet à ce dernier. Par ailleurs, dans une perspective socioconstructiviste, on reconnaît que les représentations sont socialement construites : elles se structurent au cours de processus d'échanges et d'interactions avec le groupe social de référence (Jodelet, 1989 ; Moscovici, 1989 ; Doise et coll., 1992 ; Garnier, 1999) ${ }^{1}$.

4 Selon le courant théorique structuraliste des représentations sociales (Abric, 1994 ; Flament, 1994), une représentation comporte un noyau central composé d'éléments qui sont tributaires de la trame culturelle du groupe social qui la génère. Elle comporte également des éléments périphériques qui résultent de l'expérience cumulative personnelle (idiosyncrasique) du sujet à l'égard de l'objet appréhendé; ces derniers 
éléments s'organisent en schèmes plus ou moins structurés et cohérents, qui constituent en quelque sorte des bribes explicatives de l'objet en question. C'est le noyau central, plus stable, qui détermine la signification fondamentale et l'organisation de la représentation. Quant aux éléments périphériques, ils absorbent et interprètent la nouveauté provenant de l'extérieur : les schèmes se réorganisent de façon à permettre une adaptation de la représentation aux nouvelles informations ou encore les schèmes se modifient, entraînant progressivement une remise en cause des éléments du noyau central, jusqu'à une transformation de ce dernier.

5 C'est à la lumière de ces éléments théoriques que nous nous sommes intéressés aux représentations des enseignants, comme point focal de leur apprentissage, dans le cadre d'un processus de recherche-intervention. Ce processus consistait à accompagner des enseignants déjà engagés en éducation relative à l'environnement (ERE) ${ }^{2}$ dans une démarche réflexive visant à clarifier, structurer et modéliser leur théorie et leur pratique d'ERE. D'une part, il s'agissait de développer avec ces enseignants partenaires des modèles d'intervention ${ }^{3}$ adaptés à l'école secondaire québécoise (des modèles émergeant de pratiques actuelles éprouvées), dans le but d'en proposer un répertoire à l'ensemble des enseignants; ces derniers sont, en effet, confrontés à une réforme éducative qui intègre désormais l'éducation relative à l'environnement au curriculum. Mais d'autre part, il s'agissait également d'offrir un créneau de développement professionnel aux participants de cette recherche-intervention ; dans cette perspective, la démarche a été associée à l'obtention de crédits universitaires d'études supérieures.

6 La démarche a duré plus de deux ans, de janvier 1996 à juin 1998. Le volet recherche (associé à l'intervention) s'est structuré autour de différents types d'activités : des entretiens individuels formels, des rencontres de groupe (exposés, échanges et discussions), des visites à l'école de la part de l'équipe d'encadrement, des travaux écrits (clarification des conceptions de base et réflexion sur la pratique), la tenue d'un journal de bord et des entretiens informels. En ce qui concerne l'exploration des représentations, la cueillette systématique de données s'est faite à trois moments de la démarche globale : 1) lors du premier entretien formel, 2) à mi-parcours, à l'occasion d'un travail écrit et 3) au terme de la démarche, lors d'un entretien formel.

7 Trois objets de représentation ont été considérés : l'éducation, l'environnement et l'éducation relative à l'environnement. Diverses stratégies de questionnement ont été utilisées: l'identification de mots-clés, d'images mentales, de problèmes majeurs associés à l'objet étudié ; la confrontation à une typologie de représentations usuelles (présentée en annexe 1); la confrontation critique à un répertoire de diverses théories et pratiques existantes; des questions directes (par exemple, «Selon vous, qu'est-ce que... »); la répétition ou reformulation de ces mêmes consignes et questions à des moments divers, etc. Une représentation ne se laisse, en effet, pas appréhender facilement. Il faut exploiter tout un arsenal de «sondes » pour tenter de faire émerger dans le discours, des fragments de cette représentation que le chercheur peut ensuite tenter de reconstituer (Sauvé et Garnier, 1999).

8 Au début de la recherche, plusieurs facteurs nous permettaient de penser que les enseignants formaient un groupe social particulier, susceptible de partager un certain nombre d'éléments de représentation communs (selon les critères de Rouquette, 1994): ils avaient tous une préoccupation à l'égard de l'environnement, ils avaient pris l'initiative d'intégrer l'ERE à leur pratique éducative, ils avaient développé une expertise en ce domaine, ils s'adressaient à une population du même groupe d'âge, ils 
travaillaient dans un même type d'institution, etc. Une de nos premières préoccupations a été, en ce sens, de tenter de cerner les éléments de représentation communs au groupe social «enseignants du secondaire qui pratiquent l'éducation relative à l'environnement au Québec ». Bien que le dispositif de recherche adopté n'ait pas permis de comparer ce groupe à d'autres, il a été possible de repérer des éléments d'un noyau commun aux enseignants participants ${ }^{4}$. Par exemple : l'éducation a pour mission de faciliter la transition vers l'insertion sociale ; l'environnement est un champ de préoccupation qui interpelle l'agir, de façon urgente; l'éducation relative à l'environnement correspond à un engagement, une mission.

9 Tel qu'attendu (selon le cadre théorique adopté), ces éléments du noyau central des représentations sont restés en place au cours du processus de recherche-intervention ${ }^{5}$; cette dernière a d'ailleurs eu pour effet d'accroître les interactions sociales au sein du groupe et elle était de nature à conforter les enseignants dans leurs positions fondamentales initiales.

10 Il est intéressant de noter qu'un seul des dix participants ne partageait pas, au départ, ces éléments d'un noyau commun. Les entretiens ont révélé que l'engagement initial de cet enseignant était davantage dans l'enseignement des sciences physiques de l'environnement qu'en éducation relative à l'environnement; c'est une certaine confusion des termes lors de l'enquête préliminaire qui nous avait conduits à inviter ce partenaire à faire partie du groupe de recherche. Nous verrons que, malgré (ou grâce à) cette confusion de départ, la démarche de collaboration avec cet enseignant s'est révélée des plus fructueuses.

11 Dans la perspective d'évaluer les apprentissages des enseignants participants, c'est bien davantage aux schèmes périphériques qu'aux éléments centraux que nous nous sommes intéressés: en effet, ces derniers relèvent davantage de l'expérience individuelle et se caractérisent par une plus grande plasticité. Dès les premiers entretiens, nous avons constaté que malgré les caractéristiques communes qui rattachent les enseignants participants à un même groupe, nous nous retrouvions face à des personnes très différentes, chacune ayant un parcours personnel et professionnel particulier: chaque enseignant habite un territoire géographique différent, avec ses propres problématiques environnementales; chacun enseigne dans une institution scolaire qui a sa propre dynamique ; chacun se caractérise par ses propres motivations, projets, intérêts, ambitions, etc.

12 L'analyse diachronique des éléments périphériques a permis d'observer l'évolution de certains éléments de représentation chez les participants. Trois catégories émergent de cette analyse : éléments de représentation stables, modifiés et transformés.

13 Éléments stables: les éléments périphériques sont restés sensiblement les mêmes au cours de la démarche.

14 Éléments modifiés: un changement sensible des éléments périphériques peut être observé. On peut identifier trois types de modifications :

- Type 1 : De nouveaux éléments de représentation viennent se greffer à ceux qui existaient déjà. Par exemple, chez l'un des participants, la représentation de l'éducation comme une préparation à être un "bon citoyen", s'est enrichie au fil de la recherche de l'idée de développer des acteurs sociaux compétents, qui prennent part aux décisions qui les concernent. 
- Type 2 : Des éléments déjà existants s'orchestrent différemment. Par exemple, pour l'un des enseignants, l'ERE consiste à exploiter le milieu de vie comme contexte, objet et but de l'apprentissage : il s'agit d'amener l'élève à se connaître, à connaître son milieu et à y jouer un rôle actif. Au fil de la recherche, la relation au milieu n'est plus uniquement considérée dans une perspective pédagogique, mais elle est recadrée dans une perspective éthique.

- Type 3 : Des éléments déjà existants sont davantage articulés et approfondis. Par exemple, pour l'un des participants, dont la représentation de l'environnement était initialement associée à l'idée de problèmes, les causes et conséquences de tels problèmes se sont clarifiées en cours de la recherche; un lien a été établi entre les problèmes causés par une exploitation collective abusive des ressources naturelles et ceux qui sont associés aux relations entre les humains. La notion de problème débouche désormais sur une éthique de la responsabilité.

Éléments transformés: certains éléments périphériques sont éliminés au profit de nouveaux. Par exemple, chez l'un des participants, l'éducation était considérée au départ comme un processus de transmission de connaissances pour préparer au «marché du travail». Une telle représentation s'est transformée: l'éducation est désormais conçue comme une préparation «à la vie, à l'avenir » (mais sans référence à l'insertion dans la sphère économique), où l'enseignant joue surtout le rôle de modèle (il est un adulte signifiant dont le discours et la conduite peuvent influencer les jeunes) et où il prend conscience de l'importance de la relation affective qu'il établit avec l'élève. Une telle représentation reste centrée sur l'enseignant, mais elle semble échapper à une vision exclusivement rationnelle et technologique de l'éducation (limitée à la transmission de contenus d'ordre cognitif et instrumental) dans la perspective de s'adapter aux standards d'excellence de la société de production et de consommation.

Le tableau 1 présente de façon très synthétique l'évolution de certains éléments de chacune des trois représentations analysées chez les enseignants.

Tableau 1 : Changements observés au sein des schèmes de représentation

\begin{tabular}{|l|c|c|c|}
\hline $\begin{array}{l}\text { Éléments de } \\
\text { représentation périphériques }\end{array}$ & Éducation & Environnement & ERE \\
\hline Stables & 2 & 3 & 0 \\
\hline Modifiés & 5 & 6 & 6 \\
\hline Transformés & 3 & 1 & 4 \\
\hline
\end{tabular}

On observe que chez tous les participants, il y a eu des changements de certains schèmes de représentation associés à l'éducation relative à l'environnement en cours de démarche, ce qui témoigne d'un cheminement professionnel. Chez tous les participants, on observe d'ailleurs une modification ou une transformation de schèmes pour l'une ou l'autre des représentations de l'éducation et de l'environnement. Le plus souvent, c'est la représentation de l'éducation qui montre les changements les plus importants. L'ERE, en tant que dimension de l'éducation fondamentale, suscite en effet une réflexion en profondeur sur l'éducation elle-même (Sauvé, 1997, p. 21-26).

18 Chez l'un des participants, les trois représentations se sont transformées au cours de la démarche. Il s'agit du seul enseignant qui ne semblait pas partager au départ les noyaux de représentation communs aux autres participants. L'éducation était d'abord 
associée au développement de compétences, en particulier à celles qui sont requises par la démarche scientifique; en fin de démarche, le développement de compétences est plutôt mis en lien avec l'engagement social au sein de l'école et de la communauté environnante. La représentation de l'environnement se limitait au début aux composantes biophysiques mesurables et observables du milieu ; en fin de démarche, l'environnement inclut également des éléments socioculturels et renvoie aux relations qu'entretiennent les personnes avec leur milieu de vie. Enfin, l'éducation relative à l'environnement, qui était associée au départ à une acquisition de connaissances de type scientifique sur l'environnement, devient un processus qui consiste à développer une culture de la responsabilité civique à l'égard du milieu physique et humain. Le modèle d'intervention qui a résulté de la démarche de recherche-intervention de cet enseignant, axée sur un processus d'audit environnemental de l'école, témoigne d'un développement professionnel important.

19 En revanche, d'autres participants ont montré peu de changements, conservant ou approfondissant davantage les schèmes « représentationnels » initiaux. Divers facteurs peuvent expliquer cette relative stabilité. L'un des enseignants, par exemple, avait déjà entrepris au départ de la recherche collective, un processus de réflexion en profondeur sur sa pratique en ERE, qui l'avait mené à clarifier et à systématiser sa théorie personnelle de la relation à l'environnement et de l'action éducative en ERE: le processus de recherche-intervention n'a fait que renforcer un processus réflexif déjà en cours et a simplement favorisé l'approfondissement de certains éléments de représentation préexistants. Chez d'autres participants, qui adoptaient au départ une approche pragmatique de l'ERE et qui étaient davantage préoccupés de trouver des solutions concrètes à des problèmes d'ordre stratégique, on note également moins de changements, mais pour de toutes autres raisons. Ces derniers participants sont moins enclins à valoriser et adopter une approche réflexive de leur pratique et à approfondir le volet recherche de la démarche globale de recherche-intervention. La compétence réflexive des enseignants (progressivement construite à partir de savoirs, d'habiletés et de valeurs liés à la distance critique à l'égard de sa propre pratique) apparaît à la fois comme un facteur déterminant et une résultante du développement professionnel.

Enfin, pour compléter la démarche évaluative, il importe de dépasser l'étude des changements observés, et de porter un jugement sur la valeur sur ces derniers. Cela implique l'adoption d'un référentiel, c'est-à-dire d'une théorie de l'ERE et du développement professionnel sur laquelle se fondent les jugements et sont formulées les recommandations. L'équipe d'encadrement de la recherche a effectué l'évaluation en fonction d'un cadre de référence spécifique explicite (inspiré de Sauvé, 1997). Mais, à défaut d'un référentiel collectivement construit et partagé par l'ensemble des participants, ce qui aurait exigé un processus interactif intense et à très long terme, il importait également d'inviter chacun à évaluer son propre apprentissage, en fonction de son propre cadre de référence, clarifié en cours de recherche. Une démarche d'autoévaluation a donc été entreprise, comme partie intégrante du processus de métacognition qui a présidé à l'ensemble de la démarche de recherche-intervention.

21 Il importe de signaler à cet effet que dans un tel contexte de collaboration, qui fait appel à l'investissement personnel et professionnel des acteurs et qui se prête mal à des jugements de valeur académiques exogènes, l'un des critères d'évaluation majeurs, en lien avec le cheminement d'apprentissage, a été celui de l'engagement personnel et collectif auquel ont été conviés les participants. Dans quelle mesure chacun considère- 
t-il qu'il s'est investi dans chacune des activités proposées (et négociées) au cours de la recherche intervention? Les résultats de l'ensemble du projet de la rechercheintervention, y compris ceux de la démarche évaluative, sont présentés dans Sauvé et coll. (2000).

\begin{tabular}{|c|c|c|c|c|c|c|c|}
\hline \multicolumn{8}{|c|}{$\begin{array}{l}\text { ANNEXE 1: Caractérisations des représentations-types de P’environnement } \\
\text { (Lucie Sauvé, 1996, 1997, In Sauvé et Garnier, 1999) }\end{array}$} \\
\hline & \multicolumn{7}{|c|}{ Environnement } \\
\hline & Nature & Ressource & Problème & Système & Milieu de vic & Biosphìre & Projet communautaire \\
\hline & $\begin{array}{l}\text { à apprécier } \\
\text { à respecter } \\
\text { à préserver }\end{array}$ & à gérer & à résoudre & $\begin{array}{l}\text { à comprendre } \\
\text { pour décider }\end{array}$ & $\begin{array}{l}\text { à connaitre } \\
\text { à aménager }\end{array}$ & $\begin{array}{l}\text { où vivre ensemble } \\
\text { et à long terme }\end{array}$ & où s'engager \\
\hline Mots-clés & $\begin{array}{l}\text { - nature } \\
\text { - arbres, plantes } \\
\text { animaux } \\
\text { - milicu naturel }\end{array}$ & $\begin{array}{l}\text { - l'eau, l'ais, le sol... } \\
\text { - l'́nergie } \\
\text { - la faune } \\
\text { (comme gibier) } \\
\text { - la forêt } \\
\text { (pour le bois) } \\
\text { - notre pattimoine } \\
\text { culturel }\end{array}$ & $\begin{array}{l}\text { - pollution } \\
\text { - destruction, } \\
\text { détérioration, } \\
\text { nuisances } \\
\text { - problèmes } \\
\text { écologiques }\end{array}$ & \begin{tabular}{|l} 
- écosystème \\
- équilibre \\
écologique \\
- relations \\
(écologiques, \\
environnementales)
\end{tabular} & $\begin{array}{l}\cdot \text { - ici, tout autour } \\
\text { de nous } \\
\cdot \text { - la maison, } \\
\text { le quarticr, } \\
\text { le lieu de traval } \\
\text { - mon milieu de } \\
\text { vic quotidien }\end{array}$ & $\begin{array}{l}\text { - la planète, la Terre } \\
\text { - tout } \\
\text { - l'environnement } \\
\text { global }\end{array}$ & $\begin{array}{l}\text { - notre affaire à tous } \\
\text { - notre responsabilité } \\
\text { • chose publique, } \\
\text { objet politique }\end{array}$ \\
\hline $\begin{array}{l}\text { Images } \\
\text { mentales }\end{array}$ & $\begin{array}{l}\text { - unc forêt } \\
\text { - une rivière cristalline } \\
\text { - une montagnc boisce }\end{array}$ & $\begin{array}{l}\text { - unc exploitation } \\
\text { forcstière } \\
\text { - une déchetteric } \\
\text { - unc ćolienne }\end{array}$ & $\begin{array}{l}\text { - un cours d'eau } \\
\text { pollué } \\
\text { - un tas de déchets } \\
\text { sauvages } \\
\text { - une coupe à blanc }\end{array}$ & \begin{tabular}{|l} 
- un schéma abstrait de \\
relations écologiques \\
ou ćcosystémique \\
- une forết, arec \\
l'ensemble de ses \\
composantes \\
- une ville, avec ses \\
intrants et extrants
\end{tabular} & $\begin{array}{l}\text { - unc maison et } \\
\text { son jardin } \\
\text { - un village, } \\
\text { un quarticr ou } \\
\text { un parc urbain avec } \\
\text { ses arbres, ses fleurs, } \\
\text { ses animaux familizrs } \\
\text { et son activité } \\
\text { humaine } \\
\text { - une peite ferme } \\
\text { - une carte } \\
\text { géographique à } \\
\text { petite ćchelle }\end{array}$ & $\begin{array}{l}\text { - La Terre vue de } \\
\text { lespace } \\
\text { - la miscre dans } \\
\text { un pays en } \\
\text { développement } \\
\text { - un dessin } \\
\text { représentant une } \\
\text { cosmologie } \\
\text { amérindienne }\end{array}$ & $\begin{array}{l}\text { - un groupe de } \\
\text { personnes en corvée } \\
\text { (nettoyage de rive, } \\
\text { de sites) } \\
\text { - une réunion de } \\
\text { citoyens discutant } \\
\text { démocratiquement } \\
\text { - des jardins } \\
\text { communautaires }\end{array}$ \\
\hline
\end{tabular}

ANNEXE 1: Caractérisations des représentations-types de l'environnement (suite)
(Lucie Sauvé, 1996, 1997, In Sauvé et Garnier, 1999)




\begin{tabular}{|c|c|c|c|c|c|c|c|}
\hline \multicolumn{8}{|c|}{$\begin{array}{l}\text { ANNEXE 1: Caractérisations des représentations-types de l'environnement (suite) } \\
\text { (Lucie Sauvé, 1996, 1997, In Sauvé et Garnier, 1999) }\end{array}$} \\
\hline & \multicolumn{7}{|c|}{ Environnement } \\
\hline & Nature & Ressource & Problème & Système & Milieu de vie & Biosphère & Projet communautaire \\
\hline & $\begin{array}{l}\text { à apprécier } \\
\text { à respecter } \\
\text { à préserver }\end{array}$ & à gérer & à résoudre & $\begin{array}{l}\text { à comprendre } \\
\text { pour décider }\end{array}$ & $\begin{array}{l}\text { à connaître } \\
\text { à aménager }\end{array}$ & $\begin{array}{l}\text { où vivre ensemble } \\
\text { et à long terme }\end{array}$ & où sengager \\
\hline Visée ERE & 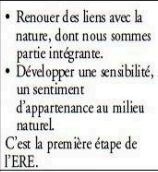 & $\begin{array}{l}\text { - Apprendre à gírer } \\
\text { l'environnement vers le } \\
\text { developpement durable, } \\
\text { le partage ́quitable } \\
\text { pour un avenir viable. }\end{array}$ & $\begin{array}{l}\text { - Développer des } \\
\text { compétences en } \\
\text { matière de résodution } \\
\text { de problèmes. } \\
\text { - Adopter des } \\
\text { comportements } \\
\text { responsables. }\end{array}$ & $\begin{array}{l}\text { - Développer la pensiée } \\
\text { systémique, pour une } \\
\text { pensée globale vers des } \\
\text { prises de décision } \\
\text { éclairées. }\end{array}$ & $\begin{array}{l}\text { - Re-découviri son milieu } \\
\text { de vive. } \\
\text { - Développer un } \\
\text { sentiment } \\
\text { dappartenance } \\
\text { à ce milieu. }\end{array}$ & \begin{tabular}{|l|} 
- Développer une \\
vision macuo- \\
environnementale. \\
- Déreloppre ure \\
conscience planéraire, de \\
type dévebppecenentale ou \\
cosmique.
\end{tabular} & \begin{tabular}{|l|} 
- Développer la praxis \\
(actiop/rélexion). \\
- Stimuler lexprit \\
critique. \\
- Valoriser lexerecice de la \\
démocratic et le travail \\
coopéracif.
\end{tabular} \\
\hline $\begin{array}{l}\text { Stratégies } \\
\text { ERE }\end{array}$ & 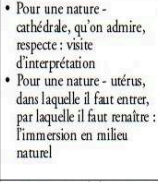 & $\begin{array}{l}\text { - campagnes d'économic } \\
\text { - incitation à la } \\
\text { récupération et } \\
\text { au reçclage } \\
\text { - audit environnemental } \\
\text { du milieu de vie }\end{array}$ & $\begin{array}{l}\text { - démarche de résolution } \\
\text { de problemes } \\
\text { - etudes de cas }\end{array}$ & $\begin{array}{l}\text { - an alyse de situations } \\
\text { environnementales avec } \\
\text { une approche sys- } \\
\text { témique } \\
\text { - exccice de prise de } \\
\text { décision }\end{array}$ & $\begin{array}{l}- \text { Étude de milieu } \\
\text { itinizariare- } \\
\text { interprétation } \\
\text { environnementale }\end{array}$ & 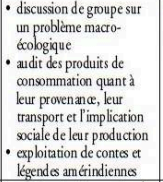 & $\begin{array}{l}- \text { la recherche-action } \\
\text { - la pédagogie de projets } \\
\text { - le forum environ- } \\
\text { nemental }\end{array}$ \\
\hline Actions & 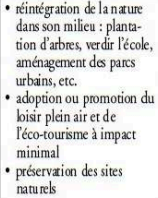 & $\begin{array}{l}\text { - contrồle des } \\
\text { prélevements } \\
\text { - Économie des ressources } \\
\text { - écoconsumérisme } \\
\text { - réduction, récupération, } \\
\text { réut ilisation, recyclage }\end{array}$ & $\begin{array}{l}\text { - projet de résolution } \\
\text { de problème } \\
\text { - nettoyage des berges, } \\
\text { testauration } \\
\text { d'ccosystemes } \\
\text { - lobbying }\end{array}$ & $\begin{array}{l}\text { - écude des systèmes } \\
\text { environnementaux } \\
\text { (ou socio- } \\
\text { environnementaux) }\end{array}$ & $\begin{array}{l}\text { - campagnes de propreté, } \\
\text { d'embenllisement } \\
\text {-jardinage cologique } \\
\text { - projets d'aménagement }\end{array}$ & 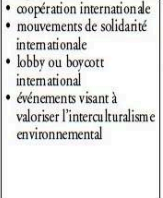 & $\begin{array}{l}\text { - projet communautaire: } \\
\text { les covées, par exemple } \\
\text { enquête collective } \\
\text { - déat démocratique }\end{array}$ \\
\hline
\end{tabular}

\section{BIBLIOGRAPHIE}

Abric, J.-C. (1994). Pratiques sociales et représentations. Paris : Presses Universitaires de France.

Doise, W., Clémence, A. et Lorenzi-Cioldi, W. (1992). Représentations sociales et analyse de données. Grenoble : Presses Universitaires de Grenoble.

Flament, C. (1994). Structure, dynamique et transformation des représentations sociales. In J.C. Abric (dir.), Pratiques sociales et représentations. Paris : Presses Universitaires de France. p. 37-58.

Garnier, C. (1999). La genèse des représentations sociales dans une perspective développementale. In M.-L. Rouquette et C. Garnier (dir.), Études sur la genèse des représentations sociales. Montréal : Éditions Nouvelles. p. 88-113.

Garnier, C. et Sauvé, L. (1999). Apport de la théorie des représentations sociales à l'éducation relative à l'environnement - Conditions pour un design de recherche. Éducation relative à l'environnement: Regards, Recherches, Réflexions, 1, 63-76.

Jodelet, D. (1989). Les représentations sociales. Paris : Presses Universitaires de France.

Machabée, L. et Sauvé, L. (2000). Les représentations des enseignants du secondaire en éducation relative à l'environnement - Une étude qualitative. In L. Sauvé et coll. (2000). Théories et pratiques de l'éducation relative à l'environnement à l'école secondaire québécoise. Tome 2 : Rapport de recherche intervention. Modèles d'intervention en ERE. Montréal : CIRADE.

Moscovici, S. (1989). Des représentations collectives aux représentations sociales. In D. Jodelet (dir.), Les représentations sociales. Paris : Presses Universitaires de France. p. 62-86.

Ponton, M. et Sauvé, L. (2000). Les représentations des enseignants du secondaire en éducation relative à l'environnement - Une étude lexicométrique. In L. Sauvé et coll. (2000). Théories et 
pratiques de l'éducation relative à l'environnement à l'école secondaire québécoise. Tome 2 : Rapport de recherche intervention. Modèles d'intervention en ERE. Montréal : CIRADE.

Rouquette, M.L. (1994). Sur la connaissance des masses. Grenoble : Presses Universitaires de Grenoble.

Sauvé, L. (1997). Pour une éducation relative à l'environnement - Éléments de design pédagogique. 2e édition. Montréal : Guérin.

Sauvé, L. et coll. (1997). L'éducation relative à l'environnement à l'école secondaire québécoise. État de la situation. Rapport d'une enquête diagnostique. Montréal : CIRADE.

Sauvé, L. et Garnier C. (1999). Une phénoménographie de l'environnement. Réflexions théoriques et méthodologiques sur l'analyse des représentations sociales. In M. Rouquette et C. Garnier (dir.), Représentations sociales et éducation. Montréal : Les Éditions nouvelles. p. 541-572.

Sauvé, L. et coll. (2000). Théories et pratiques de l'éducation relative à l'environnement à l'école secondaire québécoise. Tome 2 : Rapport de recherche intervention. Modèles d'intervention en ERE. Montréal : CIRADE.

\section{NOTES}

1. Une synthèse de l'apport de la théorie des représentations sociales à l'éducation relative à l'environnement a été présentée dans Garnier et Sauvé (1999).

2. Ces enseignants ont été repérés à la suite d'une enquête diagnostique de la situation de l'ERE à l'école secondaire québécoise (Sauvé et coll., 1997). Cette enquête avait révélé l'intérêt de leur pratique respective pour une modélisation éventuelle.

3. Un modèle d'intervention en ERE correspond à une proposition pédagogique composée de deux aspects interreliés : un cadre théorique (explicitant et justifiant une conception de l'ERE) et une démarche d'enseignement (ou d'animation) et d'apprentissage. Un tel modèle théorique validé par la pratique devient une source d'inspiration pour la pratique de l'ERE.

4. Les résultats de cette analyse des représentations sociales des enseignants sont présentés dans Machabée et Sauvé (2000) et Ponton et Sauvé (2000).

5. La recherche sur les représentations sociales montre que ces dernières se transforment très lentement : il aurait été naï d'attendre des changements fondamentaux dans le contexte si restreint de la démarche de recherche-intervention que nous avons menée.

\section{AUTEURS}

\section{LUCIE SAUVÉ}

Lucie Sauvé est professeure au département des sciences de l'éducation de l'Université du Québec à Montréal (UQAM). Au sein de cette institution, elle est également chercheure affiliée au CIRADE (Centre interdisciplinaire de recherche sur l'apprentissage et le développement en éducation) et membre de l'Institut des sciences de l'environnement. Elle assume la direction du Programme court d'études supérieures en éducation relative à l'environnement de l'UQAM et dirige des projets de coopération internationale en ERE. 


\section{LOUIS MACHABÉE}

Louis Machabée est étudiant au doctorat en sociologie, à l'Université du Québec à Montréal. Il est affilié au CIRADE et membre du Centre interuniversitaire de recherche sur la science et la technologie (CIRST) de l'UQAM. Sa recherche doctorale porte sur les logiques de l'accord en processus de restauration des écosystèmes. 